UC-34d

Issued: April 1978

\title{
Estimating Population Sizes in a Mixture of Two Radioactive Populations
}

\author{
W. A. Beyer \\ C. Qualls*
}

*Visiting Staff Member. University of New Mexico, Albuquerque, NM 87106. 
ESTIMATING POPULATION SIZES IN A MIXTUFE OF TWO RADIOACTIVE POPULATIONS

by

W. A. Beyer and C. Qualls

\begin{abstract}
The event times are observed of a mixture of two kinds of radioactive atoms, each producing alpha particles. The probability of observing an event is $p, 0<p<1$, and of missing the observation is $1-p$. A maximum likelihood stztistical method is given for estimating the size of the two populations. A second discussion assumes that the populations themselves are samples from parent populations. Bayesian methods are given for estimating parameters of the parent populations. An example is presented of an experiment involving the search for particle-bound polyneutron systems.
\end{abstract}

\title{
I. INTTRODUCTION
}

Suppose one is given a mixture of two populations, denoted by $I$ and II, of atoms in which there are $\mathrm{N}_{1}$ atoms of type $\mathrm{I}$ and $\mathrm{N}_{2}$ atoms of type II. Suppose that each population undergoes radicactive decay with known constants $\lambda_{1}$ and $\lambda_{2}$; that is, the probability that an atom of type $i$ will have decayed before time $t>0$ is $1-e^{-\lambda_{i} t}$ if, at $t i m e t=0$, the atom has not decayed. During time period $(0, \bar{t})$, the time intervals of decays of the mixture are recorded (that is, the centers of time intervals in which decay events occurred). It is given that the probability of observing a decay that occurs is $\mathrm{p}(0<\mathrm{p}<1)$. The problem is to estimate $\mathrm{N}_{1}$ and $\mathrm{N}_{2}$. In this report we give a maximum likelihosd estimate of $\mathrm{N}_{i}$ and a Bayesian probability distribution of $\mu$, when it is assumed that $\mathrm{N}_{I}$ is Poisson distributed with parameter $\mu$ and $\mathrm{N}_{2}$ is uniformly distributed over some finite interval. An application is made to an experiment by Turkevich et $a 1 .{ }^{l}$ in which an estimate of the number of polyneutrons produced is required. 
II. MAXIMUM LIKELIHOOD ESTIMATE OF POPULATION SIZES

Suppose the time interval $(0, \bar{t}]$ is subdivided by cut points $\left\{t_{i} \mid i=0,1, \ldots . k\right\}$ where $0=t_{0}<t_{1}<\ldots<t_{k}=t_{\text {. }}$ Put $q=1-p$ and $\left(t_{i-1}, t_{i}\right] \equiv I_{i}$. Let $y_{i}, l \leqslant i \leqslant k$, be the number of events (decays) that are observed in interval $i$. Suppose first that only one type of atom is present at $t=0$ and that the number of such atoms is $N$. Then the probability of observing the vector $y=\left(y_{1}, \ldots, y_{k}\right)$ is given by

$$
P(\underset{\sim}{y} \mid N)=\frac{N !}{y_{1} ! y_{2} ! \cdots y_{k} ! y !} \prod_{i=1}^{k}\left[p\left(e^{-\lambda t} i-1-e^{-\lambda t} i\right)\right] y_{i}\left(q+p e^{-\lambda \bar{t}}\right)^{y^{d}}
$$

where $\mathrm{y}^{\prime}=\mathrm{N}-\sum_{i=1}^{k} y_{i}$. In Eq. (1) the multinomial coefficient represents all ways that the set of decays of atoms can be distributed among the $k+1$ cells $I_{i}, i=1,2, \ldots, k$, and the cell $I_{k+1}$ that contains all the unobserved decays. Decays are not observed Either because the atoms decayed in $(0, \bar{t}]$ but were not observed or because they decayed after $\bar{t}$. The second factor in $E q$. (1) is the product of the probabilities of observing the $y_{i}$ events in the interval $I_{i}$. The final factor in Eq. (1) is the probability of observing the $y^{\prime}$ everits in the cell $I_{k+1}$.

Now consider the case where there are two kinds of atoms present. Let $z$ be the $k$-vector of observations. Then

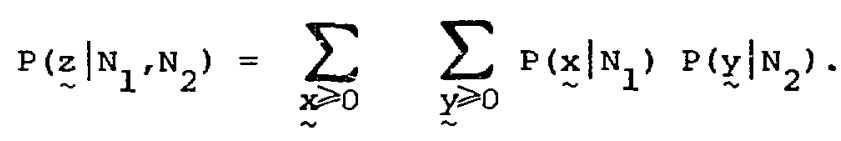

$$
\begin{aligned}
& x+y=z
\end{aligned}
$$

Put

$$
\begin{aligned}
& \sum_{i=1}^{k} y_{i}=n_{1}, \\
& \sum_{i=1}^{k} x_{i}=n_{2},
\end{aligned}
$$




$$
\begin{aligned}
& n_{1}+n_{2}=n, \\
& q_{j}=q+p e^{-\lambda_{j} \bar{t}}, j=1,2
\end{aligned}
$$

and

$$
P_{j i}=p\left(e^{-\lambda t_{i-1}}-e^{-\lambda_{j} t_{i}}\right), j=1,2,1 \leqslant i \leqslant k \text {. }
$$

Also put

$$
e_{z}\left(n_{1}\right)=\sum_{\substack{y_{i}=n_{1} \\ 0 \leqslant y \leqslant z}} \prod_{i=1}^{k} \frac{p_{i i}^{y_{i}} p_{i i}^{z_{i}-y_{i}}}{y_{i} !\left(z_{i}-y_{i}\right)}
$$

for $\max \left(0, n-N_{2}\right) \leqslant n_{1} \leqslant \min \left(n, N_{1}\right)$ and $Q_{2}\left(n_{1}\right)=0$ otherwise. Then Eq. (2) can be written as

$$
\begin{aligned}
P\left(\underset{\sim}{z} \mid N_{1}, N_{2}\right)= & \sum_{n_{1}}=\sum_{\max }^{\min \left(0, n-N_{1}\right)} \frac{N_{1} !}{\left(N_{1}-n_{1}\right) !} \frac{N_{2} !}{\left[N_{2}-\left(n-n_{1}\right)\right] !} \\
& \times q_{1}^{N_{1}-n_{1} q_{2} N_{2}-\left(n-n_{1}\right)} \underset{Q_{2}\left(n_{1}\right)}{ }
\end{aligned}
$$

for $\mathrm{N}_{1}+\mathrm{N}_{2} \geqslant \mathrm{n}$. 
The maximum, likelihood prinuiple asserts that the "best" estimate for $\mathrm{N}_{1}$ and $\mathrm{N}_{2}$ is the pair $\left(\mathrm{N}_{1}, \mathrm{~N}_{2}\right)$ that maximizes $\mathrm{F}\left(\underset{\sim}{z} \mid \mathrm{N}_{1}, \mathrm{~N}_{2}\right)$ for the observed $\underset{\sim}{2}$. For a discussion of the maximum likelihood principle, see Good ${ }^{2}$ and de Finetti. ${ }^{3}$

The fact that the quantity $q_{z}\left(n_{1}\right)$ is the coefficient of $s^{n_{1}}$ in the polynoninal

$$
\phi(s)=\prod_{1=1}^{k} \frac{\left(p_{1 i} s+p_{2 i}\right)^{z_{i}}}{z_{i} !}
$$

is the basis of a fast algorithm for computing $\mathrm{P}\left(\underset{\sim}{z} \mid \mathrm{N}_{\underline{1}}, \mathrm{~N}_{2}\right)$.

To obtain bounds on the value of $\left(\mathrm{N}_{1}, \mathrm{~N}_{2}\right)$, say $\left(\overline{\mathrm{N}}_{1}, \overline{\mathrm{N}}_{2}\right)$, that maximize $P\left(\underset{\sim}{z} \mid N_{1}, N_{2}\right)$ for a given $\underset{\sim}{z}$, it is necessary that

$$
P\left(\underset{\sim}{z} \mid \bar{N}_{1}-1, \bar{N}_{2}\right)-P\left(\underset{\sim}{z} \mid \bar{N}_{1}, \bar{N}_{2}\right) \leqslant 0
$$

and

$$
P\left(\underset{\sim}{z} \mid \overline{\mathrm{N}}_{1}, \overline{\mathrm{N}}_{2}-1\right)-P\left(\underset{\sim}{z} \mid \overline{\mathrm{N}}_{1}, \overline{\mathrm{N}}_{2}\right) \leqslant 0 .
$$

A calculation gives

$$
\begin{aligned}
& P\left(\underset{\sim}{z} \mid \bar{N}_{1}-1, \bar{N}_{2}\right)-P\left(\underset{\sim}{z} \mid \bar{N}_{1}, \bar{N}_{2}\right)=\left[-\frac{\bar{N}_{1}}{\bar{N}_{1}-\Sigma x_{i}}\left(q+p e^{-\lambda_{1} \bar{t}}\right)+1\right] \\
& \times P\left\{\underset{\sim}{z} \mid \bar{N}_{1}-1, \bar{N}_{2}\right\} \text {, }
\end{aligned}
$$


so that

$$
\bar{N}_{1} \leqslant \frac{\Sigma x_{i}}{p\left(1-e^{-\lambda_{1} \bar{t}}\right)} \leqslant \frac{\Sigma z_{i}}{p\left(1-e^{-\lambda{ }^{-\lambda}} \grave{j}^{-}\right.}
$$

Likewise,

$$
\overline{\mathrm{N}}_{2} \leqslant \frac{\Sigma_{z_{i}}}{p\left(1-e^{-\lambda_{2} \bar{E}}\right)}
$$

Also,

$$
\overline{\mathrm{N}}_{1}+\overline{\mathrm{N}}_{2} \geqslant \sum_{\mathrm{i}=1}^{\mathrm{k}} \mathrm{z}_{\mathrm{i}}
$$

III. GOODNESS-OF-FIT THEORY FOR MA:IMUM LIKELIHOOD ESTIMATE

In this section we discuss testing the null hypothesis that $\mathrm{N}_{1}=0$ based on the model discussed in Sec. $\because$. Under this hypothesis, the unknown number $\mathrm{N}_{2}$ of times of decay are independent random variables with identical exponential distributions having parameter $\lambda_{2}$. Denote these random times by $\mathrm{Y}_{1}, \mathrm{Y}_{2}, \ldots, \mathrm{Y}_{\mathrm{N}_{2}}$. Let $\Sigma$ be the set of all subsets of the indices $\left\{1,2, \ldots, \mathrm{N}_{2}\right\}$. Consider of $\Sigma$ as specifying which decays are observed in $(0, \bar{t}]$. The complement $\sigma^{\mathrm{C}}$ indicaces which decays are not observed either because they are missed with probability $q$ or because they occur after $\bar{t}$. since the probability of observing the decay during $(0, \bar{t}]$ for any given type II atom is $p\left(1-e^{-\lambda} 2^{\bar{t}}\right)$, we have a probability measure on the $2^{{ }^{2}}$ points of $\Sigma$ given by 


$$
P(\sigma)=\left(p-p e^{-\lambda_{2} \bar{t}}\right)^{n}\left(q+p e^{-\lambda_{2} \bar{t}}\right)^{N_{2}-n} \text {, }
$$

where $\mathrm{n}=|\sigma|$, the number of indices in $\sigma$.

Now the random number $N$ of observed times of decay, denoted by $x_{1}, x_{2}, \ldots, x_{1 ;}$, can be viewed as having been selected from $y_{1}, \ldots, Y_{N_{2}}$ by the random element $\sigma \varepsilon \Sigma$. Consequently, the conditional cumulative distribution function of $\mathrm{x}_{1}, \mathrm{x}_{2}, \ldots, \mathrm{x}_{\mathrm{N}}$, given $\sigma$, is

$$
\begin{aligned}
F\left(x_{1}, x_{2}, \ldots, x_{n} \mid \sigma\right) & =\prod_{i=1}^{n} P\left(y_{\sigma_{i}} \leqslant x_{i} \mid y_{\sigma_{i}} \leqslant \bar{t}\right) \\
& =\prod_{i=1}^{n} \frac{1-e^{-\lambda_{2} x_{i}}}{1-e^{-\lambda_{2} \bar{t}}} \text { for } 0<x_{i} \leqslant \bar{t}, 1 \leqslant i \leqslant n,
\end{aligned}
$$

where $n=|\sigma|$ and $\sigma_{i}$ is the $i^{\text {th }}$ member of $\sigma$ with the members of $\sigma$ considered to be ordered. The conditional probability density function of $\mathrm{x}_{1}, \mathrm{x}_{2}, \ldots, \mathrm{x}_{\mathrm{N}}$, given $\mathrm{N}=\mathrm{n}$, is

$$
\begin{aligned}
& \begin{aligned}
f\left(x_{1}, x_{2}, \ldots, x_{n} \mid n\right) & =\sum_{|\sigma|=n} f\left(x_{1}, x_{2}, \ldots, x_{n} \mid \sigma\right) P(\sigma \mid n) \\
& =\left[\prod_{i=1}^{n} \lambda_{2} e^{\left.-\lambda_{2} x_{i},\left(1-e^{-\lambda_{2} \bar{t}}\right)\right] \sum_{|\sigma|=n} P(\sigma \mid n)}\right.
\end{aligned} \\
& \text { for } 0<x_{i} \leqslant \bar{t} \text { and } 1 \leqslant i \leqslant n \text {. Note that } \sum_{|\sigma|=n} P(\sigma \mid n)=1 .
\end{aligned}
$$

6 
So we have reduced the problem of testing whether $\mathrm{N}_{1}=0$ to considering the data $x_{1}, x_{2}, \ldots, x_{N}$ conditioned on the event [N $\left.=n_{1}\right]$ as independent, identically distributed observations drawn from the distribution

$$
F(x)=\frac{1-e^{-\lambda 2^{x}}}{1-e^{-\lambda} 2^{\bar{t}}}, 0<x \leqslant \bar{t}
$$

This test can be done using the kolmogorovmSmirnov statistic

$$
D_{n}=\sup _{0<x \leqslant \bar{t}}\left|F_{n}(x)-F(x)\right|
$$

where $F_{n}$ is the empirical distribution function constructed from the data. We reject the null hypothesis that $N_{1}=0$ at a level of significance $\alpha$ if $D_{n}>d_{n, \alpha}$ where $d_{n, \alpha}$ is defined by $P\left(D_{n}>d_{n, \alpha}\right) \leqslant \alpha$. Finally, the unconditional test also satisfies $\alpha$ by the computation

$$
\begin{aligned}
P\left(D_{N}>d_{N, \alpha}\right) & =\sum_{n} P\left(D_{n}>d_{n, \alpha} \mid N=n\right) P(N=n) \\
& \leqslant \alpha \sum_{n} P(N=n)=\alpha .
\end{aligned}
$$

\section{A BAYESIAN ESTIMATE}

A second mathematical model of the two-population problem will now be discussed. This model, which is used later, assumes that each of the population sizes is a random variable. The second population size $\mathrm{N}_{2}$ is a random variable uniformly distributed on $L$ to $L+\eta$. The first population size $\mathrm{N}_{1}$ is selected at random from a Poissor. distribution with parameter $\mu$, with "efficiency" $\gamma, 0<\gamma<1$, so that $\mathrm{N}_{1}$ is a random variable with the distribution 


$$
\frac{e^{-\gamma \mu}(\gamma \mu)^{k}}{k !}, \quad k=0,1,2, \ldots
$$

where $Y$ is given. The Poisson parameter is obtained from considering a compound Poisson distribution. See Feller, ${ }^{4}$ p. 171, problem 27, and p. 288.

Assume these distributions for $\mathrm{N}_{1}$ and $\mathrm{N}_{2}$ are superimposed upon the random experiment described in Sec. II. If the random experiment is repeated $\ell$ times with vector outcomes $z_{1}, z_{\sim}, \ldots, z_{\sim} \ell^{\prime}$ then the probability of obtaining the vectors $z_{i}$ for a given $\mu$ is given $b_{i}$

$$
\begin{gathered}
f \underset{\sim j}{z}, 1 \leqslant i \leqslant \ell \mid \mu)=\frac{1}{(n+1)^{\ell}} \prod_{i=1}^{\ell} \sum_{N_{1}^{i}=0}^{\infty} \sum_{N_{2}^{i}=L}^{L+n} P\left(z_{1} \mid N_{1}^{i}, N_{2}^{i}\right) \\
x e^{-\gamma_{i} \mu \frac{\left(\gamma_{i} \mu\right)^{N}}{N_{1}^{i}}} .
\end{gathered}
$$

Now suppose one assumes that $\mu$ has a probability distribution $p(\mu)$ on $(0, \infty)$. Then it follows from Bayes' formula that the posterior distribution of $\mu$ for given vectors $\underset{\sim i}{z}$ is

$$
g\left(\mu \mid z_{i^{\prime}}, 1 \leqslant i \leqslant \ell\right)=\frac{f\left(z_{i^{\prime}}, 1 \leqslant i \leqslant \ell \mid \mu\right) p(\mu)}{\int_{0}^{\infty} f\left(z_{i^{\prime}}, 1 \leqslant i \leqslant \ell \mid \bar{\mu}\right) p(\bar{\mu}) d \bar{\mu}}
$$

If one puts $\mathrm{p}(\mu)=1 / \mathrm{a}$ for $0<\mu<\mathrm{a}$ and $\mathrm{p}(\mu)=0$ for $\mu>\mathrm{a}$ and then takes the limit as $a \rightarrow \infty$, one obtains

$$
g\left(\mu \mid{\underset{\sim}{z}}_{i}, 1 \leqslant i \leqslant \ell\right)=\frac{f\left(\sim_{i}, 1 \leqslant i \leqslant \ell \mid \mu\right)}{\int_{0}^{\infty} f\left({\underset{\sim}{z}}_{i}, 1 \leqslant i \leqslant \ell \mid \bar{\mu}\right) d \bar{\mu}},
$$

provided the denominator in Eq. (6) converges. 
We now prove this convergence. In the following, $\sum_{1,2}$ is defined

by

$$
\sum_{1,2}=\sum_{N_{1}=0}^{\infty} \sum_{N_{2}=L}^{L+n}
$$

One has for each $a, 0<a<\infty$, and for some $j, 1 \leqslant j \leqslant \ell$,

$$
\begin{aligned}
& \left.\int_{0}^{a} f(\underset{\sim}{z}, 1 \leqslant i \leqslant \ell \mid \bar{\mu}) d \bar{\mu} \leqslant \int_{0}^{a} f \underset{\sim j}{z} \mid \bar{\mu}\right) d \bar{\mu}
\end{aligned}
$$

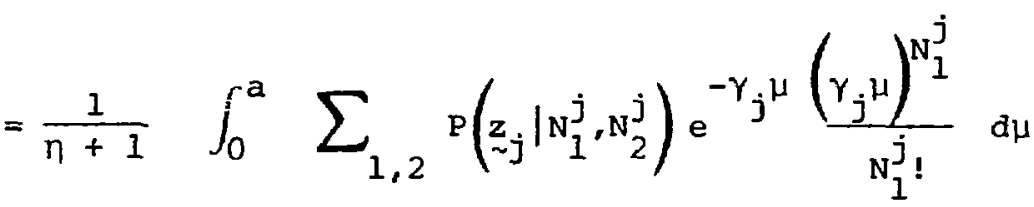

$$
\begin{aligned}
& \leqslant \frac{1}{\gamma_{j}} \sum_{j, 2} P\left(z_{\sim j} \mid N_{1}^{j}, N_{2}^{j}\right) \\
& =\frac{1}{\gamma} \sum_{\substack{1,2 \\
N_{1}+N_{2} \geqslant n}} \sum_{n_{1}=\max \left(0, n_{-n}\right)}^{\min \left(n, N_{1}\right)} \frac{N_{1} !}{\left(N_{1}-n_{1}\right) !} \frac{N_{2} !}{\left(N_{2}-\left(n-n_{1}\right)\right] !}
\end{aligned}
$$

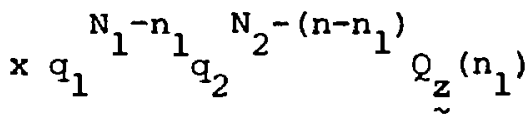

(suppressing the index $j$ )

$$
=\frac{1}{\gamma} \sum_{N_{2}=\mathrm{L}}^{L+n} \sum_{n_{1}=}^{n} \frac{N_{2} !}{\max \left(0, n-N_{2}\right)} \frac{q_{1}^{-n_{1}} N_{q_{2}}-\left(n-n_{1}\right)}{\left[N_{2}-\left(n-n_{1}\right)\right] !} q_{2}^{\left(n_{1}\right)}
$$

9 


$$
x \sum_{N_{1}=n_{1}}^{\infty} \frac{N_{1} !}{\left(N_{l}-n_{l}\right) !} q_{1}^{N_{1}}<\infty,
$$

because $0<q_{1}<1$. So

$$
\int_{0}^{a} f\left(z_{i}, 1 \leqslant i \leqslant \ell \mid \bar{\mu}\right) d \bar{\mu}
$$

is bounded and monotonically increasing as $\mathrm{a} \rightarrow \infty$ and therefore has a limit.

V. DESCRIPTION OF EXPERIMENT

The objective of an experiment recently performed by Turkevich et al.. partly diagrammed in Fig. I, was to measure the number of particle-bound polyneutron systems $x_{n}$ (for short: polyneutrons) produced in the reaction

$800-\mathrm{MeV}$ protons + target $\rightarrow \mathrm{x}_{\mathrm{n}}+$ fragments.

The target may be, in various experiments, boron carbide, lead, or uranium. The following discussion depends on restricting attention to polyneutrons with $x \geqslant 4$.

The detection of polyneutrons in this experiment is somewhat conplicated. The target is partly surrounded by a lead detector of $\sim 4 \mathrm{~kg}$ of metallic lead, $52 \%$ being $208 \mathrm{pb}$. The polyneutrons that are produced may react with ${ }^{208} \mathrm{pb}$ in the lead detector according to

$$
x_{n}+{ }^{208} P b+{ }^{212} P b+(x-4) n
$$

The ${ }^{212} \mathrm{~Pb}$ then undergoes the following decay chains with indicated half-life times and, in the second case, the probabilities are given for the two branches: 

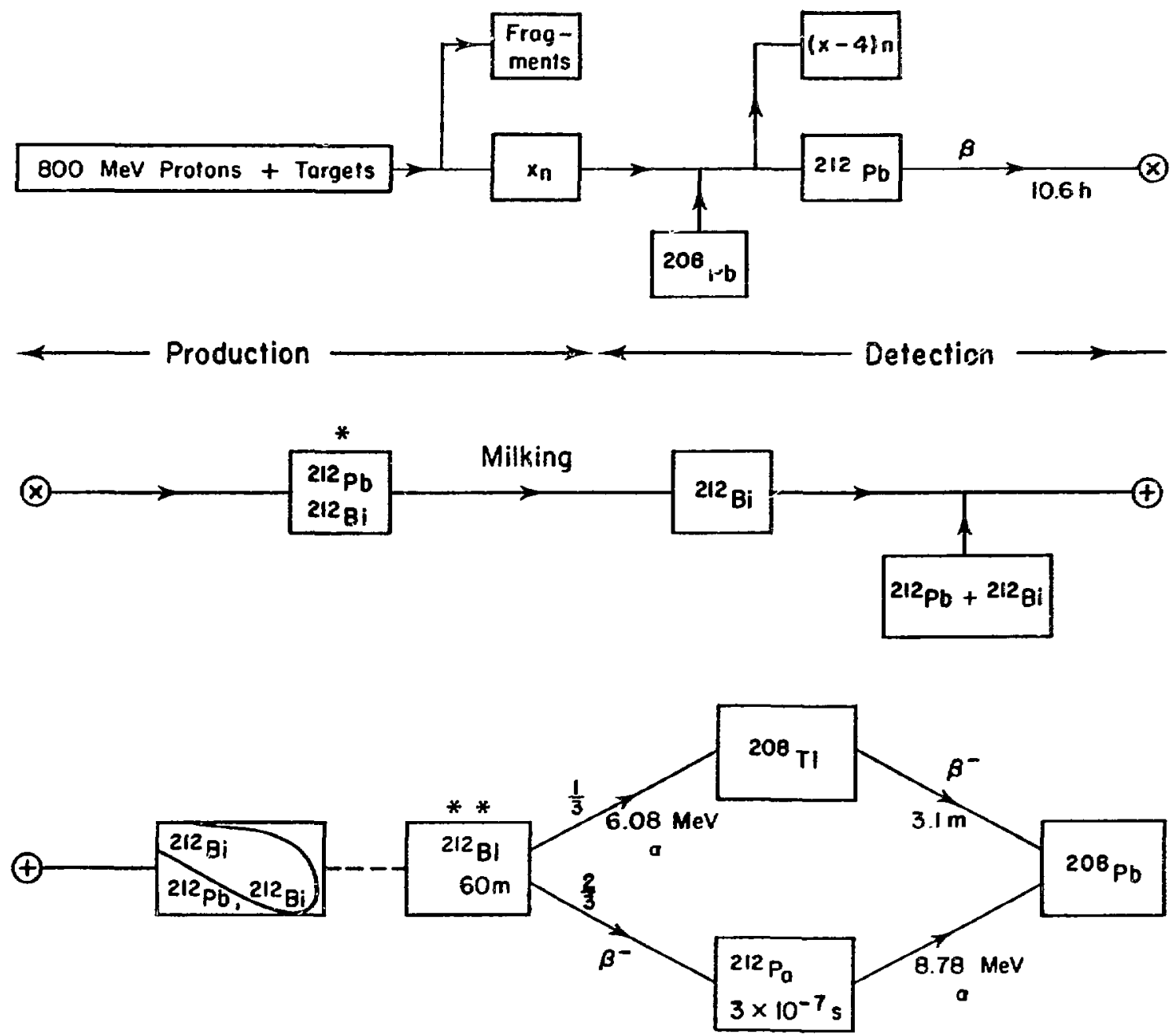

Fig. 1.

Decay chain in experiment.

${ }^{212} \mathrm{~Pb} \rightarrow{ }^{212} \mathrm{Bi}+\mathrm{e}^{-}+\bar{v}(10.6 \mathrm{~h})$,

$212{ }_{\mathrm{Bi}}^{21{ }^{3}}-\mathrm{PO}_{\mathrm{PO}}+\mathrm{e}^{-}+\bar{v}(60.6 \mathrm{~min})$,
$208_{\mathrm{Tl}}+\alpha(6.08 \mathrm{MeV})(60.6 \mathrm{~min} ;$,

$$
{ }^{212} \mathrm{Po} \rightarrow{ }^{208} \mathrm{~Pb}+\alpha(8.78 \mathrm{MeV})(\sim \mu \mathrm{s}),
$$

and

$$
208_{\mathrm{Tl}}+{ }^{208} \mathrm{~Pb}+\mathrm{e}^{-}+\bar{\nu}(3.1 \mathrm{~min}) .
$$


After exposure of the target to protons, the lead detector is dissolved. The solution containing ${ }^{21.2} \mathrm{~Pb}$ and ${ }^{212} \mathrm{Bi}$ at the location in Fig. 1 marked (*) is called a "cow." A few mg $\left(\sim 10^{19}\right.$ atoms) of ${ }^{209} \mathrm{Bi}$ are added to the solution and the mixture of ${ }^{209} \mathrm{Bi}$ and ${ }^{212} \mathrm{Bi}$, with tracer amcunts of ${ }^{207} \mathrm{Bi}$ (long-lived gamma emitter), are removed chemically from this mixture. The removal process is called "milking." A fraction 1 - $\gamma$ of the atoms of the mixture is lost during the milking. The fraction $\gamma$ is called the efficiency and can be determined by recovery of the macroscopic amounts of ${ }^{209}$ Bi added or from recovery of the gamma ralioactivity of ${ }^{207} \mathrm{Bi}$. In the process of milking, atoms of ${ }^{212} \mathrm{~Pb}$ and its daughter ${ }^{212} \mathrm{~B} . \dot{\mathrm{i}}$ are introduced by contamination from the atmosphere, reagents, and glassware. These two contamination products are in radioactive equilibrium. The isolated sample of bismuth, marked $(* *)$ in Fig. 1 , is put in an alpha counter and the 8.78-MeV alphadecays, selected with good discrimination from cther energy alpha particles, are observed for a period of time. Because of counter geometry, the fraction of alpha-decays recorded is about 0.3. After a milking, the number of $212_{\mathrm{Bi}}$ atoms in the "cow" again increases from zero to some number in rarioactive equilibrium with the $212 \mathrm{~Pb}$ present.

Because of the short half-life of ${ }^{212} \mathrm{po}$ and because the $6.08 \mathrm{MeV}$ alpha-decays are not counted, the reactions (9) to (11) can be replaced in this study by

$$
212 \mathrm{Bi} \rightarrow{ }^{208} \mathrm{~Pb}+\mathrm{e}^{-}+\alpha(8.78 \mathrm{MeV}), \mathrm{p}=2 / 3,(60.6 \mathrm{~min})
$$

Thereafter, ${ }^{212} \mathrm{Bi}$ refers to those ${ }^{212} \mathrm{Bi}$ with $8.78 \mathrm{MeV} \alpha$-particle progeny: Consider the sample marked $(* *)$ in Fig. 1. This sample contains $212_{B i}$ and ${ }^{212} \mathrm{~Pb}$. There are two sources for the ${ }^{212} \mathrm{Bi}$. One source is the chain which commenced with the reaction $x_{n}+{ }^{208} \mathrm{~Pb}$. The other source is the contaminate ${ }^{212} \mathrm{Bi}$ in decay equilibrium with its mother ${ }^{212} \mathrm{~Pb}$. It follows that for each decay of the mother contaminate ${ }^{212} \mathrm{~Pb}$ there is a decay of the dauginter contaminate ${ }^{212} \mathrm{Bi}$. Thus the decay of the ${ }^{212} \mathrm{Bi}$ havirig the $\mathrm{x}_{\mathrm{n}}$ as an ancestor appears to have a half-life of $60.6 \mathrm{~min}$ and the decay of the contaminate $\mathrm{Bi}$ appears to have a half-life of $10.6 \mathrm{~h}$. (For further discussion, see Appendix A). This implies that $\lambda_{1}=0.01144$ decays $/ \mathrm{min}$ and $\lambda_{2}=0.00109$ decays/min. 
The objective is then to determine from the time history of the a]nha-particle count, the number of (apparent) 10.6-h $212 \mathrm{Bi}$. toms present in the sample and the number of $60.6-\mathrm{min} 212 \mathrm{Bi}$ atoms present in the sample. The lead decector, labeled $(*)$ in Fig. 1 , was milked sequentially three times in the experiment reported by Turkevich et al. Each of the samples was placed under a counter for a length of time. Table I gives the time histories of the $8.78-\mathrm{MeV}$ events. The values are the centers of $15-\mathrm{min}$ intervals in which an event was registered. Time is recorded in minutes from the beginning of the counting.

TABLE I

TIME HISTORIES OF $8.78-\mathrm{MEV}$ FVENTS FOR THE THREE MILKINGS

\begin{tabular}{|c|c|c|c|}
\hline & $I$ & II & III \\
\hline & 22 & 53 & 8 \\
\hline & 235 & 98 & 52 \\
\hline & 250 & 114 & 177 \\
\hline & 359 & 221 & 318 \\
\hline & 406 & 304 & 349 \\
\hline & 545 & 315 & 364 \\
\hline & 792 & 488 & 456 \\
\hline & 1157 & 719 & 487 \\
\hline & & 796 & 502 \\
\hline & & 1010 & 517 \\
\hline & & 1086 & 793 \\
\hline & & & 884 \\
\hline & & & 914 \\
\hline & & & 1083 \\
\hline Number of events & 8 & 11 & 14 \\
\hline $\begin{array}{l}\text { Termination of } \\
\text { counting }\end{array}$ & 1500 & 1350 & 1500 \\
\hline $\begin{array}{l}\text { Efficiency } \gamma \\
\text { of milking }\end{array}$ & 0.47 & 0.68 & \\
\hline
\end{tabular}


VJ. APPLICATION OF MAXIMUM LIKELIHOOD ESTIMATION TO THE EXPERIMENT

Ir this section we apply the mathematical model of Sec. II to the data given in Sec. To tobtain joint estimates of $\mathrm{N}_{1}$ and $\mathrm{N}_{2}$.

The maximum likelihood estimates of $\mathrm{N}_{1}, \mathrm{~N}_{2}$, together with the maximum value of $P\left\{\underset{\sim}{z} \mid N_{2}, N_{2}\right\}$ given by Eq. (2), are presented in Table II for each of. the three milkings. Here $\mathrm{N}_{1}$ is the estimated number of ${ }^{212}$ Bi atoms with $x_{n}$ as ancestor present after milkings and at the start of measurement $(t=0)$, and $\mathrm{N}_{2}$ is the estimated number of contaminate ${ }^{212} \mathrm{Bi}$ atoms present at $t=0$. The column in Table II marked "combined data" is calculated by assuming there is a milking in which the counts are obtained by interleaving the counts from each of the milkings; thus: $8,22,52,53,98,114,177,221,235$, etc. The time of termination of the counting in the combined data is $\bar{t}=1350$. Note that the efficiency $\gamma$ does not appear in these calr' 'ations because $\mathrm{N}_{1}$ refers to atoms after milking.

The estimates of $\mathrm{N}_{1}$ indicate that a small number of polyneutrons might have been present in the milkings. Of course these es:-imates are subject to statistical fluctuations and it would be desirable to place upper and lower bounds on these estimates. This is mathematically difficult, but the goodness-of-fit result of Sec. III does say something about the lower bound. In Sec. VII a different model is used to obtain an upper bound.

Applying the Kolmogorov-Smirnov goodness-of-fit test ${ }^{5}$ when one assumes $N_{1}=0$ gives the results listed in Table III. This table indicates that the model of Sec. II with no polyneutrons present $\left(\mathrm{N}_{1}=0\right)$ is consistent

TABLE II

VALUES OF $\left(\mathrm{N}_{1}, \mathrm{~N}_{2}\right)$ THAT MAXIMIZE $\mathrm{P}\left(\underset{\sim}{2} \mid \mathrm{N}_{1}, \mathrm{~N}_{2}\right)$

\begin{tabular}{|c|c|c|c|c|}
\hline & $I$ & II & III & Combined Data \\
\hline $\mathrm{N}_{1}$ & 1 & 2 & 2 & 4 \\
\hline $\mathrm{N}_{2}$ & 48 & 68 & 85 & 200 \\
\hline$P\left(\underset{\sim}{z} \mid N_{1}, N_{2}\right)$ & $2.9 \times 10^{-12}$ & $7.0 \times 10^{-15}$ & $1.1 \times 10^{-17}$ & $4.6 \times 10^{-29}$ \\
\hline
\end{tabular}


TABLE III

K-S GOODNESS-OF-FIT RESULTS

$\begin{array}{lcccc} & \text { I } & \text { II } & \text { III } & \text { Combined } \\ \begin{array}{lllll}\text { Kolmogorov-Smirnov } \\ \text { Statistic } D_{n}\end{array} & 0.194 & 0.169 & 0.179 & 0.116 \\ \begin{array}{l}\text { Critical values } \\ \text { for } \alpha=0.10\end{array} & 0.411 & 0.352 & 0.314 & 0.21\end{array}$

with the data from the experiment. Consequently $\mathrm{N}_{1}=0$ is no doubt. the lower bound one would obtain by any reasonable procedure.

It seems reasonable that the data are also consistent with the model of sec. II where $\mathrm{N}_{1}$ and $\mathrm{N}_{2}$ are the maximum likelihood estimates. This statement is based on two arguments. First, estimates of $\mathrm{N}_{1}$ are close to $\mathbf{N}_{1}=0$, and therefore the fit should be similar. Second, it is reasonable to expect the full model where the number $\mathrm{N}_{1}$ of polyneutrons is not restricted to zero to fit the data as well as the restricted model does.

VII. APPLICATION OF BAYESIAN ESTIMATION TO THE EXPERIMENT

The second mathematical model (Sec. IV) is applied to escimating the number of ${ }^{212} \mathrm{Bi}$ atoms having $\mathrm{x}_{\mathrm{n}}$ as an ancestor as follows. As is stated in Sec. IV, the ${ }^{212} \mathrm{Bi}$ atoms present in the lead detector are the daughters of ${ }^{212} \mathrm{~Pb}$ atoms. Hence the number of ${ }^{212} \mathrm{Bi}$ atoms having $\mathrm{x}_{\mathrm{n}}$ as an ancestor present at any given time is a random variable. We wish to show that this random variable is Poisson distributed. Suppose initially w atoms of type ${ }^{212} \mathrm{~Pb}$ and zero daughter atoms ${ }^{212} \mathrm{Bi}$ are present in the lead detector. Let $\lambda_{1}$ be the decay constant of the ${ }^{212} \mathrm{~Pb}$ and $\lambda_{2}$ the decay constant for ${ }^{212} \mathrm{Bi}$. What is the probability for a single atom $212^{2} \mathrm{~Pb}$ to have decayed to ${ }^{212} \mathrm{Bi}$ and not further before time $t$ ? Divide the interval $(0, t)$ into sub-intervals of length $\Delta \tau$ each. Then the probability that the above occurs is

$$
\sum_{\text {all intervals }}\left[e^{-\lambda_{1} \tau}-e^{-\lambda_{1}(\tau+\Delta \tau)}\right] e^{-\lambda_{2}(t-\tau-\Delta \tau)}
$$




$$
\begin{aligned}
& \rightarrow \lambda_{1} \int_{0}^{t} e^{-\lambda_{1} \tau} e^{-\lambda_{2}(t-\tau)}, \quad \text { as } \Delta \tau \rightarrow 0 \\
& =\frac{\lambda_{1}}{\lambda_{2}-\lambda_{1}}\left(e^{-\lambda_{1} t}-e^{-\lambda_{2} t}\right) .
\end{aligned}
$$

Call the last quantity $f(t)$. The probability of $i$ atoms at $t$ being in the form ${ }^{212} \mathrm{Bi}$ is ther.

$$
\left(\begin{array}{l}
w \\
i
\end{array}\right)[f(t)]^{i}[1-f(t)]^{w-i}
$$

For $w$ large, $f(t)$ small, and $w f(t)$ moderate, this is approximated by the Poisson distribution (Feller, ${ }^{4}$ p. 153)

$$
\frac{[w f(t)]^{i}}{i !} e^{-w f(t)}
$$

(Also, cf. Feller, ${ }^{4}$ p. 424, problem 11, where it assumed that $w$ is constant). Therefore, it is reasonable to assume the number $\mathrm{N}$ of ${ }^{212} \mathrm{Bi}$ atoms with ${ }_{\mathrm{n}}$ as ancestor has a Poisson distribution $e^{-\mu} \mu^{k} / k !, k=0,1,2, \ldots$, where $\mu=w f(t)$ is a parameter to be estimated. Note that $\mu$ refers to the number of atoms present before the milkings.

It is assumed that the time between milkings is short enough that $\mu$ does not change and long enough that the amount of ${ }^{212} \mathrm{Bi}$ reaches a steady state before each of the three milkings. It will be further assumed that the number of contaminate atoms $\mathrm{N}_{2}^{i}$ is uniformly distributed between 30 and 120 . (A small number of subsequent dry-run experiments where no polyneutrons were created shows that this uniform distribution assumption on $\mathrm{N}_{2}$ is not unreasonable.) For a given $\mu$, the probability of producing three given vectors $\mathbf{z}_{i}$ ' $i=1,2,3$ of observations is then

$$
E\left(z_{1}, z_{2}, z_{3} \mid \mu\right)=
$$




$$
\frac{1}{91^{3}} \prod_{i=1}^{3} \sum_{N_{1}^{i}=0}^{\infty} \sum_{N_{2}^{i}=30}^{1.20} P\left(z_{i} \mid N_{1}^{i}, N_{2}^{i}\right) e^{-\gamma_{i} \mu}\left(\gamma_{i} \mu\right)^{N_{1}^{i}} / N_{1}^{i} !
$$

For the three milkings and corresponding counts reported in Sec. $V$, the value of $\mu$ that maximizes Eq. (12) (maximum likelihood value) is given by $\mu=1.59$ and the value of Eq. (12) is $2.66 \times 10^{-17}$.

To compare this estimate of $\mu=1.59$ to the estimates given in Table II for the first mathematical model, it is necessary to multiply by the efficiencies for each of the three milkings. The results rounded to the nearest integer are not inconsistent with the results in Table III.

The estimate; of Sec. IV and App. B give the final estimate

$$
\int_{0}^{\infty} f\left(z_{1}, z_{2}, z_{3}^{z} \mid \mu\right) d \mu=3.48 \times 10^{-13}
$$

Figure 2, the graph of the probability distribution $g\left(\mu \mid \sum_{\sim 1}, z_{2}, z_{3}\right)$ for $\mu$ :Eq. (6)] for vectors $\underset{\sim}{\mathbf{z}}$ determined experimentally as discussed in Sec. $v$. shows that the probability that $0 \leqslant \mu \leqslant 17.3$ equals 0.9 . One may interpret this as a $90 \%$ interval estimate of $\mu$.

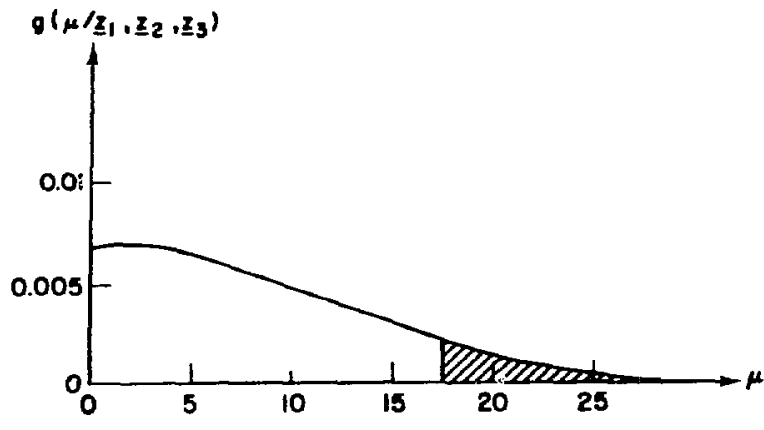

Fig. 2 .

The probability distribution $g\left(\mu / z_{l}\right.$ $\left.z_{2}, z_{3}\right)$ as a function of $\mu$. $\quad$ s 908 confidence interval is $(0,17.3)$.
VIII. CONCLUSIONS

A mixture of two populations of radioactive atoms is observed for a finite time by observing their alpha particle decays. The problem is that of estimating the sizes of these two populations, given the two decay constants. With some given probability, alpha particle emission events may occur, but not be observed. Two methods of estimation are presented: maximum likelihood estimation and Bayesian estimation. 
In the maximum likelihood estimation, a formula [Eq. (2)] is derived, which expresses the probability $P\left\{\underset{\sim}{2} \mid N_{1}, N_{2}\right\}$ of a vector $z$ of observations for given population sizes $\mathrm{N}_{1}$ and $\mathrm{N}_{2}$ of the two populations. In the Bayesian estimation, the second population size $\mathrm{N}_{2}$ is assumed to be a random variable uniformly distributed on the positive integers between $L$ and $L+n$. The first population size $\mathrm{N}_{1}$ is assumed to be selected from a Poisson distribution

$$
e^{-\gamma \mu} \frac{(\gamma \mu)^{k}}{k !}, k=0,1,2, \ldots
$$

where $\mu$ is uniformly distributed in a generalized sense on $(0, \infty)$ and $\gamma$, $0<Y<1$, is an efficiency that depends on the experiment. The probability distribution on $\mu$, for a given set of $l$ experiments with observation vectors $z_{1},{\underset{\sim}{2}}_{2}, \ldots, z_{2}$ is given by Eq. (6).

These two models are applied to experimental results in a search for particle-bound polyneutron systems reported by Turkevich et al. The two models gave consistent answers. The maximum likelihood estimate of $\mathrm{N}_{1}$ was $\mathrm{N}_{1}=4$. The Bayesian estimate $\mu$ is 1.59 for the combined three experiments and $90 \%$ of the posterior distribution of $\mu$ is in the interval $(0,17.3)$. It is emphasized that the maximum likelihood estimate of $\mathrm{N}_{1}=4$ is of the total number of polyneutron indicators obtained in all the milkings at the box marked $(* *)$ in Fig. 1 , while the Bayesian estimate $\mu=1.59$ is of the mean number of polyneutron indicators available to any one of the three milkings at the box marked (*) in Fig. 1. To compare the estimates of the two models in the report, one can consider the combined estimate of $\mathrm{N}_{1}=4$ to be an estimate of the parameter $\mu\left(\gamma_{1}+\gamma_{2}+\gamma_{3}\right)$. Knowing the efficiences $\gamma_{i}$, the maximum likelihood model can then be used to estimate $\mu=2 . \ddot{5}$, while the Bayesian modei estimates $\mu=1.59$.

Some open mathematical problems should be mentioned: (1) extend the goodness-of-fit results of Sec. III, which were restricted to the case $N_{1}=0$, to the full model and (2) determine if a confidence interval for $\mathrm{N}_{1}$ can be derived without using Bayesian techniques. 
ACKNOWLEDGMENT

The authors thank Nicholas Metropolis, Anthony Turkevich, and

Michael S. Waterman for advice.

REFERENCES

1. A. Turkevich, J. R. Cadieux, J. Warren, T. Economou, J. La Rosa, and H. R. Heydegger, "Search for Particle-Bound Polyneutron Systems," Fhys. Rev. Lett. 38, 1129-1131 (1977).

2. I. J. Good, The Estimation of Probabilities: An Essay on Modern Bayesian Methods (MIT Press, Cambridge, 1965).

3. B. de Finetti, Probability, Induction and Statistics (John Wiley \& Sons, Inc., New York, 1972).

4. W. Feller, An Introduction to Probability Theory and its Applications, 3rd ed. (John Wiley \& Sons, Inc., New York, 1968).

5. W. H. Beyer, Ed., Handbook of Tables for Probability aná Statistics, 2nd ed., (The Chemical Rubber Co., Cleveland, 1958). 


\section{APPENDIX A}

DECAY OF PARIICLES INITIAILY IN RADIOACTIVE EQUILIBRIUM

The proof of the statemeat on p. 10, Sec. V, is as follows. Recall that there are two chains in the sample, both of which involve the decay of $212_{\mathrm{Bi}}$.
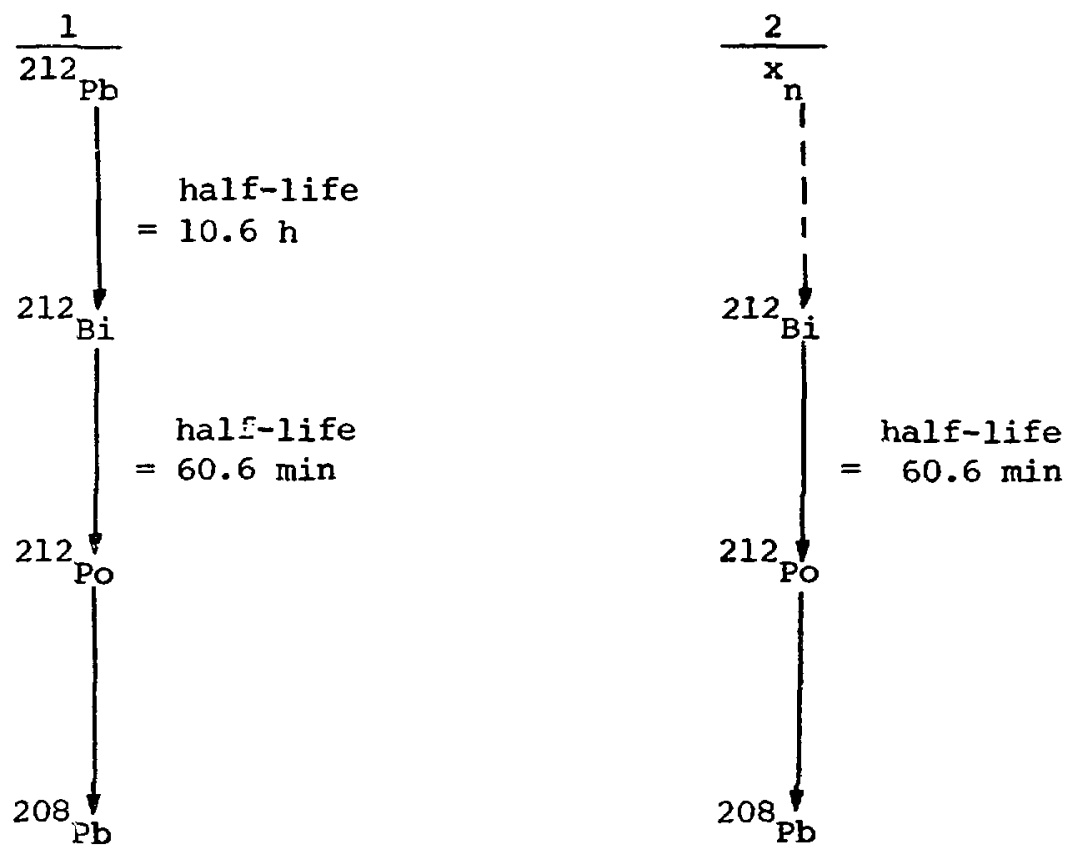

In chain 1, the amounts of ${ }^{212} \mathrm{~Pb}$ and $212_{\mathrm{Bi}}$ present initially are such that they are in radioactive equilibrium. In chain 2 , only ${ }^{212} \mathrm{Bi}$ is present initially. Let $\mathrm{Pb}^{j}(t)$ and $\mathrm{Bi}^{j}(t), j=1$ and 2 , be the amount of ${ }^{212} \mathrm{~Pb}$ and ${ }^{212} \mathrm{Bi}$ present in chain $j$. In chain 1 ,

$$
\frac{\mathrm{d} \mathrm{Pb}^{1}}{\mathrm{dt}}=\lambda_{1} \mathrm{~Pb}^{1}
$$


and

$$
\frac{d \mathrm{Bi}^{1}}{\mathrm{dt}}=\lambda_{1} \mathrm{~Pb}^{1}-\lambda_{2} \mathrm{Bi}^{1}
$$

In chain 2 ,

$$
\frac{\mathrm{dB \textrm {Bi } ^ { 2 }}}{\mathrm{dt}}=\lambda_{2} \mathrm{Bi}^{2}
$$

or

$$
B i^{2}(t)=B i^{2}(0) e^{-\lambda} 2^{t}
$$

In chain 1, because of initial equilibrium,

$$
\frac{\mathrm{dBi}}{\mathrm{dt}} \quad(0)=0 \text {, }
$$

so that

$$
\mathrm{Bi}^{1}(0)=\frac{\lambda_{1}}{\lambda_{2}} \mathrm{~Pb}^{1}(0)
$$

Now

$$
\mathrm{Pb}^{1}(t)=\mathrm{Pb}^{1}(0) \mathrm{e}^{-\lambda_{1} \mathrm{t}}
$$

and

$$
\frac{\mathrm{dBi}}{\mathrm{dt}}=\lambda_{1} \mathrm{~Pb}^{1}(0) \mathrm{e}^{-\lambda_{1} \mathrm{t}}-\lambda_{2} \mathrm{Bi}^{1}
$$

or

$$
\frac{\mathrm{dB} \mathrm{i}^{1}}{\mathrm{dt}}+\lambda_{2} \mathrm{Bi}^{1}=\lambda_{1} \mathrm{~Pb}^{1}(0) \mathrm{e}^{-\lambda_{1} \mathrm{t}}
$$


or (suppressing the superscript "l")

$$
\begin{aligned}
B i(t) & =e^{-\lambda_{2} t} \lambda_{1} P b(0) \int_{0}^{t} e^{\left(\lambda_{2}-\lambda_{1}\right) \tau} d \tau+e^{-\lambda_{2} t} B i(0) \\
& =e^{-\lambda_{2} t} \lambda_{1} P b(0)\left[\frac{e^{\left(\lambda_{2}-\lambda_{1}\right) t}-1}{\lambda_{2}-\lambda_{1}}\right]+e^{-\lambda_{2} t} B i(0) \\
& =B i(0) e^{-\lambda_{1} t}\left[1+\lambda_{1} t+\frac{\left(\lambda_{1}-\lambda_{2}\right)\left(1+\lambda_{1}-\lambda_{2}\right) t^{2}}{2 !}+\ldots\right] .
\end{aligned}
$$

Thus for small $\lambda_{2} t$,

$$
B i^{1}(t) \cong B i^{1}(0) e^{-\lambda{ }^{t}}
$$

A comparison of Eqs. $(A-1)$ and $(A-2)$ shows more precisely what is asserted in the statement that the decay of ${ }^{212}{ }_{B i}$ having $x_{n}$ as an ancestor appears to have a half-life of $60.6 \mathrm{~min}$ and the decay of the contan nate ${ }^{212} \mathrm{Bi}$ appears to have a half-life of $10.6 \mathrm{~h}$.

\section{A. PENDIX $B$}

ES TIMAT $\Xi$ OF REMAINDER IN INFINITE INTEGRAL

Numerical integration is used to estimate the integral appearing in Eq. (6) when $\ell=3$ :

$$
\int_{0}^{\infty} f\left(\underset{\sim 1}{z_{1}}, \underset{\sim 2}{z},{\underset{\sim}{3}}_{3} \mid \mu\right) d \mu .
$$


The integral is estimated by

$$
\int_{0}^{\infty} f\left(\underset{\sim 1}{z_{1}}, \underset{\sim 2}{z_{2}}, \underset{\sim 3}{z_{3}} \mid \mu\right) d \mu \simeq \sum_{i=1}^{\bar{i}} f\left(\underset{\sim 1}{z_{1}}, \underset{\sim 2}{z_{2}}, \underset{\sim 3}{z_{3}} \mid \mu_{1}\right) \Delta \mu+\int_{A}^{\infty} f\left(\underset{\sim 1}{z_{1}},{\underset{\sim}{z} 2}_{2}{\underset{\sim}{z}}_{3} \mid \mu\right) d \mu,
$$

where $\Delta \mu=0.1, \mu_{i}=0.1 i(i=1,2, \ldots, \bar{i})$, and $A=0.1 \bar{i}+0.05$. Choose $A$ so that $\int_{A}^{\infty}$ is small. A value for $A$ is estimated as follows. Define

$$
\left(\mathrm{P}_{2} \mathrm{P}_{3}\right)^{\max }=\max _{\mathrm{N}_{1}, \mathrm{~N}_{2}} \mathrm{P}\left(\underset{\sim 2}{z_{2}} \mid \mathrm{N}_{1}, \mathrm{~N}_{2}\right) \max _{\mathrm{N}_{1}, \mathrm{~N}_{2}} \mathrm{P}\left(\underset{\sim 3}{\left(\mathrm{z}_{3}\right.} \mid \mathrm{N}_{1}, \mathrm{~N}_{2}\right)
$$

Define $\sum_{1,2}^{i}$ as in Eq. (7) but with $\mathrm{N}^{\mathbf{i}}$ replacing $\mathrm{N}$. Then

$$
\begin{aligned}
& \int_{A}^{\infty} f\left(z_{I^{\prime}}, z_{\sim 2}, z_{3} \mid \mu\right) d \mu \\
& =\frac{1}{(n+1)^{3}} \int_{A}^{\infty} \prod_{i=1}^{3} \sum_{1,2}^{i} P \underset{\sim i}{P\left(z_{1} \mid N_{1}^{i}, N_{2}^{i}\right)} e^{-\gamma_{i}^{\mu}} \frac{\left(\gamma_{i} \mu\right)^{N_{1}^{i}}}{N_{1}^{i} !} d \mu \text {. }
\end{aligned}
$$

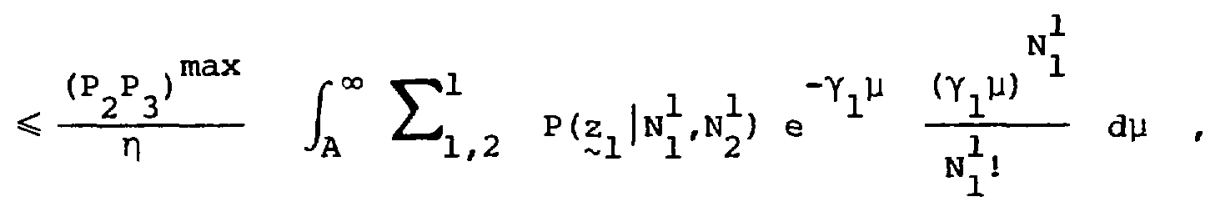

$$
\begin{aligned}
& \leqslant \frac{\left(\mathrm{P}_{2} \mathrm{P}_{3}\right)^{\max }}{\eta} \sum_{1,2}^{1} \underset{\sim}{\mathrm{P}\left(\mathrm{z}_{1} \mid \mathrm{N}_{1}^{1}, \mathrm{~N}_{2}^{1}\right)} \frac{1}{\mathrm{~N}_{1}^{1} !} \int_{\mathrm{A}}^{\infty} e^{-\gamma_{1} \mu}\left(\gamma_{1} \mu\right)^{\mathrm{N}_{1}^{1}} \mathrm{~d} \mu . \\
& =\frac{\left(\mathrm{P}_{2} \mathrm{P}_{3}\right)^{\max }}{\eta} \sum_{1,2} \mathrm{P}\left(\underset{\sim}{\left.\mathrm{z} \mid \mathrm{N}_{1}, \mathrm{~N}_{2}\right)} \frac{1}{\mathrm{~N}_{1} ! \gamma} \int_{\gamma A}^{\infty} e^{-\mathrm{x}} \mathrm{x}^{\mathrm{N}_{1}} d x .\right.
\end{aligned}
$$


(suppressing "1")

$$
=\frac{\left(P_{2} P_{3}\right)^{\max } e^{-\gamma A}}{\eta \gamma} \sum_{1,2} P\left(\underset{\sim}{z} \mid N, N_{2}\right) \frac{(\gamma A)^{N}}{N !}\left[1+\frac{N}{\gamma A}+\frac{N(N-I)}{(\gamma A)^{2}}+\ldots+\frac{N !}{(\gamma A)^{N}}\right] \text {, }
$$

(whiere $\mathrm{N}=\mathrm{N}_{1}$ )

$$
\begin{aligned}
& =\frac{\left(P_{2} P_{3}\right)^{\max } e^{-\gamma A}}{n \gamma} \sum_{1,2} P\left(\underset{\sim}{z} \mid N, N_{2}\right)(\gamma A)^{N}\left[\frac{1}{N !}+\frac{1}{(N-1) ! \gamma A}+\ldots+\frac{1}{(\gamma A)^{N}}\right] \\
& <\left(P_{2} P_{3}\right)^{\max } \frac{e^{-\gamma A}}{\eta \gamma} \sum_{1,2} \underbrace{}_{\sim}\left(\underset{z}{z} \mid N, N_{2}\right)(\gamma A)^{N} \frac{(N+1)}{(\gamma A)^{r-1}(N-Y+1) !}
\end{aligned}
$$

$$
\begin{aligned}
& \{\text { whero } r=\max (0,[N-\gamma A])\} \\
& =\left(P_{2} P_{3}\right)^{\max } \frac{e^{-\gamma A}}{n \gamma} \sum_{1,2} \sum_{n_{1}=\max \left(0, n_{-N}\right)}^{\min \left(n_{2}, N\right)} \frac{N !}{\left(N-n_{1}\right) !\left[N_{2}-\left(n-n_{1}\right) !\right]} \\
& x q_{1}^{N-n_{1}} q_{2}^{q_{2}^{-\left(n-n_{1}\right)}} \quad q_{z}\left(n_{1}\right) \frac{(\gamma A)^{N}(N+1)}{(\gamma A)^{r-1}(N-r+1) !} \\
& =\frac{\left(P_{2} P_{3}\right)^{\max } e^{-\gamma A}}{n \gamma} \sum_{N_{2}=L}^{L+\eta 1} \sum_{n_{1}=\max \left(0, n-N_{2}\right)}^{n} \frac{N_{2} !}{\left[N_{2}-\left(n-n_{1}\right)\right] !}
\end{aligned}
$$

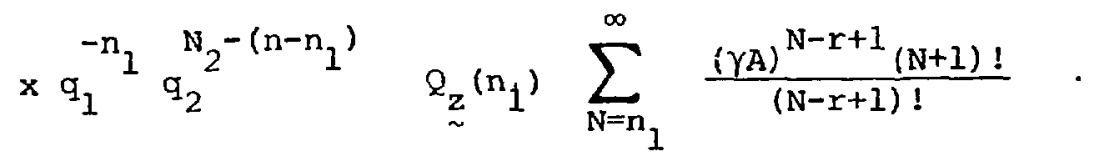


The infinite series $i$, estimated as follows.

$$
\begin{aligned}
& \sum_{N=n_{1}}^{\infty} \frac{q_{1}^{N}}{\left(N-n_{1}\right) !} \frac{(\gamma A)^{N-r+1}}{(N-r+1) !}(N+1) ! \\
& \leqslant \frac{\left(\gamma^{A}\right)^{\gamma A+2}}{[\gamma A] !} \sum_{N=n_{1}}^{\infty}(N+1)(N)(N-1) \ldots\left(N-n_{1}+1\right) q_{1}^{N} \\
& \leqslant \frac{(\gamma A)^{\gamma A+2}}{[\gamma A] !} \sum_{N=n_{1}}^{\infty}(N+1)^{n} q_{1} .
\end{aligned}
$$

The series $\sum_{\mathrm{N}=\mathrm{n}_{1}}^{\infty}(\mathrm{N}+1)^{\mathrm{n}_{1}} \mathrm{q}_{1}^{\mathrm{N}}$ is convergent because $0<\mathrm{q}_{1}<1$ and its remainder may be estimated. 